\title{
Mitosis-specific phosphorylation of Mis18a by Aurora B kinase enhances kinetochore recruitment of polo-like kinase 1
}

\author{
Minkyoung Lee ${ }^{1, *}$, Ik Soo Kim ${ }^{1, *}$, Koog Chan Park², Jong-Seo Kim, Sung Hee Baek ${ }^{1}$, \\ and Keun II Kim ${ }^{2}$ \\ ${ }^{1}$ Creative Research Initiatives Center for Chromatin Dynamics, Department of Biological Sciences, Seoul National University, \\ Seoul 08826, South Korea \\ ${ }^{2}$ Department of Biological Sciences, Cellular Heterogeneity Research Center, Sookmyung Women's University, Seoul 04310, \\ South Korea \\ ${ }^{3}$ Center for RNA Research, Institute for Basic Science, Department of Biological Sciences, Seoul National University, Seoul \\ 08826, South Korea \\ "These authors contributed equally to this work
}

Correspondence to: Keun Il Kim, email: kikim@sookmyung.ac.kr Sung Hee Baek, email: sbaek@snu.ac.kr

Keywords: Mis18a; Aurora B kinase; PLK1; mitosis-specific phosphorylation; polo box domain

Received: August 06, $2017 \quad$ Accepted: October 28, $2017 \quad$ Published: November 27, 2017

Copyright: Lee et al. This is an open-access article distributed under the terms of the Creative Commons Attribution License 3.0 (CC BY $3.0)$, which permits unrestricted use, distribution, and reproduction in any medium, provided the original author and source are credited.

\section{ABSTRACT}

Mis18a, a component of Mis18 complex comprising of Mis18a, Mis18 $\beta$, and M18BP1, is known to localize at the centromere from late telophase to early G1 phase and plays a priming role in CENP-A deposition. Although its role in CENP-A deposition is well established, the other function of Mis18a remains unknown. Here, we elucidate a new function of Mis18a that is critical for the proper progression of cell cycle independent of its role in CENP-A deposition. We find that Aurora B kinase phosphorylates Mis18a during mitosis not affecting neither centromere localization of Mis18 complex nor centromere loading of CENP-A. However, the replacement of endogenous Mis18a by phosphorylation-defective mutant causes mitotic defects including micronuclei formation, chromosome misalignment, and chromosomal bridges. Together, our data demonstrate that Aurora B kinase-mediated mitotic phosphorylation of Mis18a is a crucial event for faithful cell cycle progression through the enhanced recruitment of polo-like kinase 1 to the kinetochore.

\section{INTRODUCTION}

Accurate segregation of duplicated chromosomes is crucial for daughter cells to have one copy of each chromosome during cell division. To complete accurate segregation, the chromosome should be condensed properly and mitotic spindle should bind to kinetochore bioriented [1]. The kinetochore is formed on a chromosomal locus called centromere that is composed of DNA segments and histone proteins containing centromerespecific H3 variant, CENP-A [2]. Mis18 complex (Mis18 $\alpha$, Mis18 $\beta$ and M18BP1) in higher eukaryote is a critical factor for the recruitment of newly synthesized CENP-A to the centromere at early G1 phase [3, 4].

Previously, it has been reported that deletion of Mis $18 \alpha$ in mice causes embryonic lethality as well as defect in epidermal stratification, which are accompanied with CENP-A loss at the centromere and defects in chromosome segregation [3, 5]. Mis 18 complex localizes to the centromere from telophase to early G1 phase of cell cycle prior to the CENP-A deposition to centromere $[4,6]$. Phosphorylation of M18BP1 is involved in the regulation of the timing of centromere localization and licensing function of Mis18 complex. CDK1/2mediated phosphorylation of M18BP1 on multiple sites blocks its interaction with Mis18 $\alpha /$ Mis18 $\beta$ and hence centromere localization during $\mathrm{S} / \mathrm{G} 2 / \mathrm{M}$ phases, whereas phosphorylation of M18BP1 by PLK1 at early G1 phase facilitates centromere localization of Mis18 complex and its licensing function $[7,8]$.

Among mitotic kinases, Aurora serine/threonine kinases work crucially during mitosis. Aurora A 
kinase locates pericentrosome and regulates mitotic spindle assembly, centrosome separation and G2/M transition at the beginning of mitosis [9, 10]. Aurora $\mathrm{B}$ kinase locates innercentromere from prometaphase to metaphase regulating chromatin modification and chromatid separation, and relocates to midzone for cytokinesis [11]. Phosphorylation of Aurora B targets in the innercentromere participates in spindle checkpoint and regulates the microtubule-kinetochore interaction $[12,13]$. Dephosphorylation of the Aurora B targets gives strong tension between microtubule and kinetochore allowing the cells to go to anaphase [1].

Recently, Aurora B kinase-PLK1-MCAK (mitotic centromere-associated kinesin) axis has been shown to be required for accurate chromosome segregation [14]. At the kinetochore, Aurora B kinase activates PLK1 by phosphorylation and the activated PLK1 in turn phosphorylates MCAK, which is essential for accrurate chromosome segregation with its increased microtubule depolymerase activity. Inhibition of either Aurora B kinase or PLK1 reduces MCAK phosphorylation on PLK1 target sites and induces formation of impolar mitotic spindle and the chromatin bridges. Interestingly, PLK1 is also needed for the full activation of Aurora B kinase at the beginning of prometaphase. Aurora B kinase, Survivin, INCENP, and borealin are members of chromosomal passenger complex (CPC) and Survivin phosphorylation by PLK1 elicits Aurora B kinase acitivity around kinetochore [15]. Thus, the cooperation between Aurora B kinase and PLK1 is a very important biological process for accurate chromosome segregation.

In this study, we report that Aurora B kinase phosphorylates Mis18 $\alpha$ during mitosis, specifically at prometaphase which is critical for the faithful chromosome segregation. During prometaphase, microtubule dynamically interacts with kinetochore for the proper attachment and the process is regulated by Aurora B kinase and PLK1. Notably, we found Mis 18 $\alpha$ phosphorylation by Aurora B kinase is important for the recruitment of PLK1 to the kinetochore and for preventing the mitotic defects.

\section{RESULTS}

\section{Mis18 $\alpha$ is phosphorylated during mitosis by Aurora B kinase}

Although Mis $18 \alpha$ has been shown to function as a licensing factor for the recruitment of newly synthesized CENP-A to centromere at G1 phase, whether Mis $18 \alpha$ is involved in the processes of cell division cycle has not been investigated. As Mis $18 \alpha$ protein level is not changed through the cell cycle, we anticipated that posttranslational modification of Mis $18 \alpha$ might act as a signal for the regulating Mis $18 \alpha$ function. Therefore, we analyzed whether Mis $18 \alpha$ is phosphorylated during cell cycle progression by the mitotic kinases that actively regulate mitosis. HeLa cells stably expressing Flag-Mis $18 \alpha$ were mitotically synchronized by nocodazole treatment and the phosphorylation level of Mis18 $\alpha$ was analyzed. Interestingly, we detected increased phosphorylation level of Mis $18 \alpha$ from the mitotic cell extracts comparable to the H3S10 phosphorylation, a mitotic marker (Figure 1A). Consistently, Mis $18 \alpha$ phosphorylation increased at mitotic phase after release from $\mathrm{G} 1 / \mathrm{S}$ cell cycle synchronization by double thymidine block (Figure 1B), confirming mitosis-specific phosphorylation of Mis18 $\alpha$. We next screened for potential kinases that are responsible for Mis $18 \alpha$ phosphorylation during mitosis. Among several mitotic kinases tested, only Aurora B kinase was able to phosphorylate Mis18 $\alpha$ (Figure 1C). We also found the increased binding between Mis $18 \alpha$ and Aurora B kinase during mitosis (Figure 1D and 1E), which matches well with the phosphorylation pattern of Mis $18 \alpha$. Furthermore, the kinase dead (KD) mutant of Aurora B kinase failed to phosphorylate Mis $18 \alpha$ (Figure 1F), indicating that Aurora B kinase activity is crucial for Mis $18 \alpha$ phosphorylation.

Next, we searched for the phosphorylation site by Aurora B kinase in Mis $18 \alpha$, which is recognized by the consensus sequence, $[\mathrm{R} / \mathrm{K}]-\mathrm{X}-[\mathrm{S} / \mathrm{T}]$, and found that Mis $18 \alpha$ contains only one serine residue that matches with the consensus sequence in both mouse (Ser13) and human (Ser36) (Figure 1G). LC-MS/MS analysis of Mis18 $\alpha$ from mitotically synchronized $293 \mathrm{~T}$ cells confirmed Ser36 as the phosphorylation site during mitosis (Supplementary Figure 1). Thus, we generated phosphorylation-specific antibody against the peptide of Mis $18 \alpha$ and the resulting antibody detected phosphorylated form of peptide much stronger than non-phospho peptide control (Figure 1H). We then evaluated the specificity of our phospho-specific Mis $18 \alpha$ antibody. The antibody detected a specific band corresponding to Mis $18 \alpha$ only in nocodazoletreated cell extracts and $\lambda$-phosphatase (a Ser/Thr/Tyr phosphatase) treatment abolished the signal (Figure 1I). In addition, the antibody efficiently detected the increased phosphorylation of Mis $18 \alpha$ WT in nocodazolearrested cells. However, the expression of Mis18 $\alpha$ SA, a mutant Mis $18 \alpha$ containing alanine substitution of Ser36, did not give rise to any significant signal (Figure 1J). In an attempt to clarify the time of Mis $18 \alpha$ Ser36 phosphorylation with this new phospho-specific antibody, the antibody generated the strongest signal during mitosis in consistency with $\mathrm{H} 3 \mathrm{~S} 10$ phosphorylation (Supplementary Figure 2A). Furthermore, synchronization of cells by mitotic drugs other than nocodazole, such as monastrol and taxol, increased phosphorylation of Mis $18 \alpha$ (Supplementary Figure 2B). In an in vitro kinase assay with bacterially expressed Mis $18 \alpha$, purified active Aurora B phosphorylated wild-type Mis18 $\alpha$ but not Mis $18 \alpha$ SA (Figure 1K). Mis $18 \alpha$ phosphorylation increased in mitotic cells, but decreased as the cells exited mitosis (Figure 1L). MG132, which induces metaphase arrest by inhibiting 
APC-mediated proteolysis [17], maintained Mis $18 \alpha$ phosphorylation in parallel with $\mathrm{H} 3 \mathrm{~S} 10$ phosphorylation, although the cells were released from nocodazolemediated arrest (Figure 1M). Concurrently, the binding between Aurora B kinase and Mis18 $\alpha$ increased during mitosis and decreased as the cells exited mitosis, but not under APC block, in parallel with Mis $18 \alpha$ phosphorylation pattern (Figure 1N). Moreover, the treatment of cells with Aurora B kinase inhibitor, Hesperadin [16] diminished the phosphorylation of Mis $18 \alpha$ induced by mitotic arrest (Figure 1O), indicating that Aurora B kinase is responsible for the phosphorylation of Mis $18 \alpha$. Taken together, these results indicate that the phosphorylation of Mis $18 \alpha$ is a mitosis-specific event mediated by Aurora B kinase.

\section{Mis18 $\alpha$ phosphorylation is necessary for faithful mitotic division}

To find out the role of Mis18 $\alpha$ phosphorylation, we generated HeLa cells stably expressing shRNAresistant form of Mis18 $\alpha$ WT $\left(\mathrm{WT}^{\mathrm{R}}\right)$ or Mis18 $\alpha$ SA $\left(\mathrm{SA}^{\mathrm{R}}\right)$. The knockdown of endogenous Mis18 $\alpha$ was achieved by lentiviral infection of pLKO-shMis $18 \alpha$ just before experiment. The infection of cells with lentivirus reduced the level of endogenous Mis $18 \alpha$ efficiently (Supplementary Figure 3). The specific phosphorylation of reconstituted Mis $18 \alpha$ proteins was validated by immunoblot analysis (Figure 2A). With these reconstituted cell lines, we then checked the cell division and interestingly, the number of cells showing misaligned chromosomes (white arrow) at metaphase increased 2.5fold in Mis18 $\alpha$ SA-reconstituted cells compared with Mis $18 \alpha$ WT-reconstituted cells (Figure 2B). In addition, either the chromatin bridges or lagging chromatids and micronuclei (white arrow) increased approximately two folds in Mis18 $\alpha$ SA-reconstituted cells than Mis18 $\alpha$ WTreconstituted cells (Figure 2C).

To verify whether Mis $18 \alpha$ phosphorylation is necessary for mitosis, we next examined the mitotic defects in Mis 18aff/ESR-Cre MEFs in which endogenous Mis $18 \alpha$ can be depleted by the treatment with tamoxifen (Figure 2D). Mis 18a ff/ESR-Cre MEFs were reconstituted with either Mis18 $\alpha$ WT or Mis18 $\alpha$ SA, and we detected mitotic phosphorylation of Mis $18 \alpha$ only in Mis $18 \alpha$ WTreconstituted MEFs, but not in Mis18 $\alpha$ SA-reconstituted MEFs (Figure 2E). Interestingly, the depletion of endogenous Mis $18 \alpha$ induced an increase of aneuploidy in Mis $18 \alpha$ SA-reconstituted MEFs compared with Mis $18 \alpha$ WT-reconstituted MEFs; especially the population of cells containing the number of chromosome over $4 \mathrm{~N}$ were increased in Mis18 $\alpha$ SA-reconstituted MEFs compared with Mis $18 \alpha$ WT-reconstituted MEFs (Figure 2F and Supplementary Figure 4). This is consistent with the previous studies that showed Aurora B depletion or overexpression increasing aneuploidy [18, 19]. Furthermore, the depletion of endogenous Mis $18 \alpha$ increased the number of the chromatin bridges or lagging chromatids by four times when compared with untreated control cells (Figure 2G). While Mis18 $\alpha$ WTreconstitution significantly reduced the chromatin bridges or lagging chromatids formation in Mis $18 \alpha$-deficient MEFs, Mis $18 \alpha$ SA-reconstitution failed to do so (Figure $2 \mathrm{G}$ and $2 \mathrm{H}$ ). In addition, the micronuclei formation and chromosome misalignment also increased in Mis $18 \alpha$ deficient MEFs and these were not recovered by Mis $18 \alpha$ SA-reconstitution similarly to the chromatin bridges or lagging chromatids formation (Figure 2G,2I and 2J). However, CENP-A dots were intact indicating that these mitotic defects in Mis18 $\alpha$ SA-reconstituted MEFs are independent of CENP-A deposition. Taken together, mitotic phosphorylation of Mis $18 \alpha$ by Aurora B kinase is necessary both for the faithful segregation of chromosome.

\section{Mis18 $\alpha$ phosphorylation is not required for CENP-A loading}

Since Mis $18 \alpha$ plays a role in CENP-A loading process as a licensing or priming factor [4, 7], we questioned whether the phosphorylation of Mis $18 \alpha$ by Aurora B kinase is important for this function. Therefore, we first checked the Mis18 complex formation which is essential for CENP-A loading process and found that Mis $18 \alpha$ SA has little or no defect in the binding with either Mis $18 \beta$ or M18BP1 (Figure 3A and 3B). Furthermore, Mis $18 \alpha$ WT began to show as dots at centromere from anaphase and stayed there until G1 phase (Figure 3C) as reported previously [4]. Mis18 $\alpha$ SA showed the similar pattern with WT, indicating that Mis $18 \alpha$ phosphorylation at Ser36 by Aurora B kinase is not crucial for its centromere localization.

Next, we examined whether the phosphorylation of Mis $18 \alpha$ is required for the CENP-A loading process by adopting and modifying the experimental scheme that was used to show the prerequisite function of Mis $18 \alpha$ for the centromere loading of newly synthesized CENP-A [4]. HeLa cells stably expressing siRNA-resistant form of Mis $18 \alpha$ WT or Mis $18 \alpha$ SA were transfected with siRNA against Mis $18 \alpha$ to get rid of endogenous Mis $18 \alpha$ and then transfected with GFP-CENP-A as shown in the scheme of Figure 3D. After 24 and 9 hours of siRNA and GFPCENP-A transfection, respectively, GFP-CENP-A dots were clearly observed at late telophase of both WT and SA-expressing cells (Figure 3D).

We applied an alternative scheme to confirm that Mis $18 \alpha$ phosphorylation is unrelated to CENP-A loading process. In this experiment, we could check the effect of prolonged Mis18 $\alpha$ knockdown and the reconstitution Mis $18 \alpha$ WT or Mis $18 \alpha$ SA on CENP-A loading process. HeLa cells were infected with lentivirus expressing shRNA against Mis $18 \alpha$ and reconstituted with either Mis $18 \alpha$ WT or Mis $18 \alpha$ SA as shown in the scheme of Figure 3E. Cells were synchronized by treating with monastrol and 
A

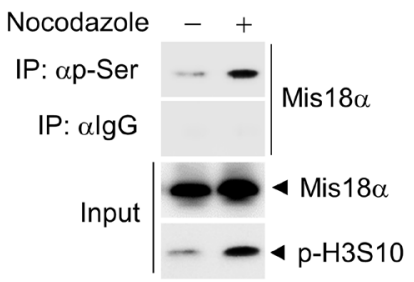

D

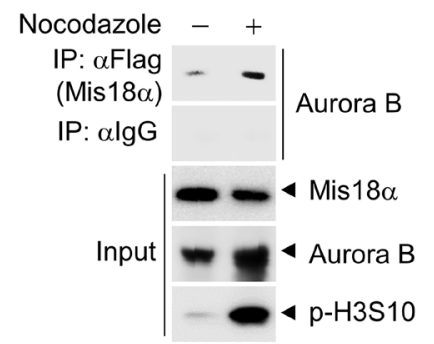

G

Aurora B kinase sequence (R/K)-x-(S/T)

M. musculus 7 LLEKRLㅗㅌEDSSRY 19 H. sapiens 30 LLGKRLㅌEDSSRH 42

H

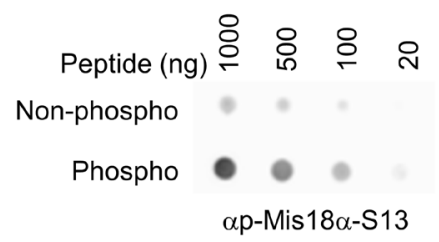

Double thymidine block

E

Double thymidine block

$\begin{array}{lllllll}\text { Release (h) } & 5 & 7 & 9 & 10 & 11 & 13\end{array}$
C
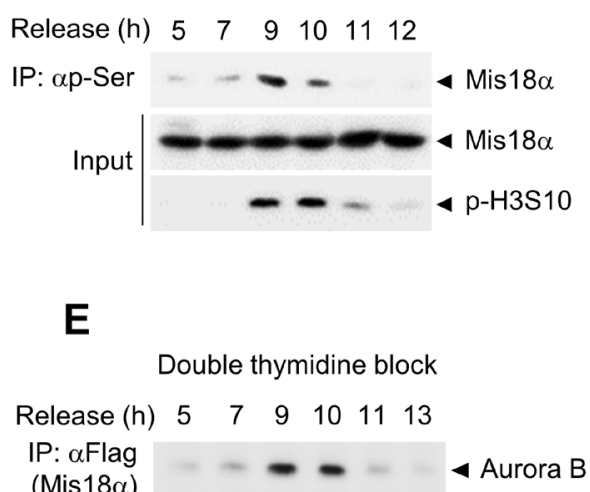

- - - - - Mis $18 \alpha$

Input $=828<44$ Aurora B

$-\ldots$ - $-\mathrm{H} 3 \mathrm{~S} 10$

$\mathbf{F}$

IP: ap-Ser $\quad 4$ Mis18
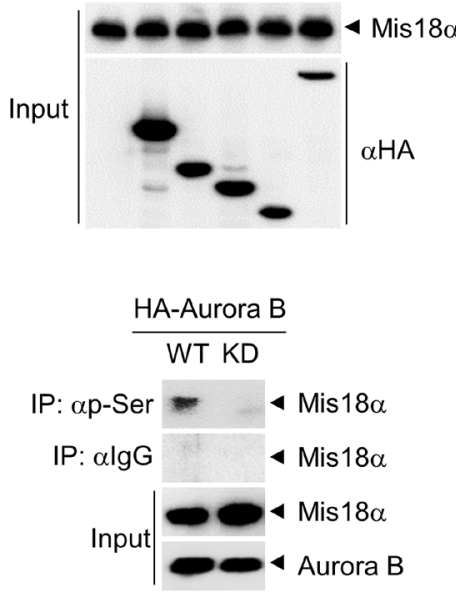

I

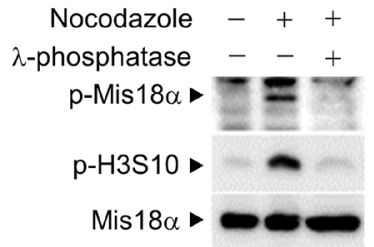

$\mathbf{L}$

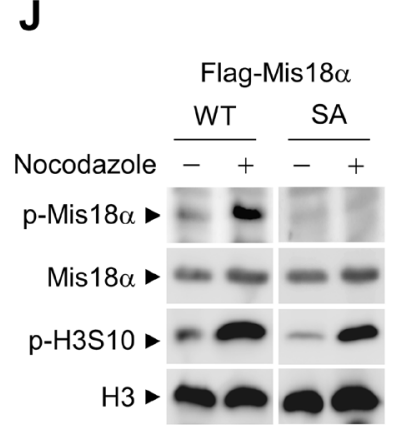

K
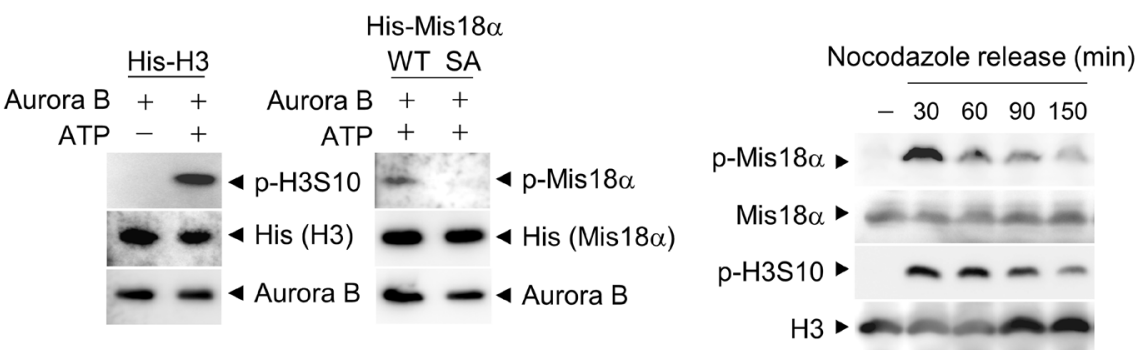

0

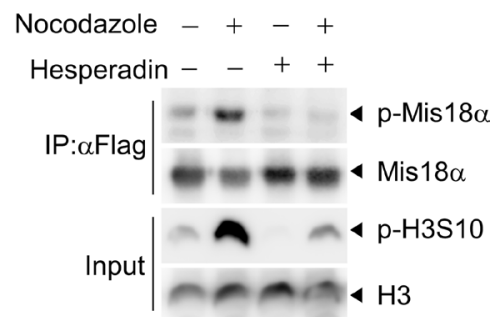

Figure 1: Mis18 $\alpha$ is phosphorylated during mitosis by Aurora B kinase. (A) HeLa cells stably expressing Flag-Mis $18 \alpha(\mathrm{HeLa} /$ Flag-Mis 18 $\alpha$ ) were synchronized by nocodazole treatment. Cell extracts were subjected to immunoprecipitation (IP) with an antibody against phosphorylated-serine (p-Ser) followed by immunoblotting with anti-Flag antibody. Phosphorylation of 10th serine residue of histone H3 (p-H3S10) was used as a mitosis indicator. (B) HeLa/Flag-Mis18 $\alpha$ cells were synchronized at G1/S by double thymidine block and released into indicated time points and were analyzed as in (A). (C) Mitotic kinases were transfected into HeLa/Flag-Mis18 $\alpha$ cells and cell extracts were applied to IP with the anti-p-Ser antibody followed by immunoblotting with anti-Flag antibody. (D) Mitotically arrested $\mathrm{HeLa} /$ Flag-Mis $18 \alpha$ cells with nocodazole treatment were applied for IP with anti-Flag antibody and detected with anti-Aurora B antibody. (E) HeLa/Flag-Mis18 $\alpha$ cells prepared as in B were used for IP assay with anti-Flag antibody and detected with anti-Aurora B antibody. 
(F) HeLa/Flag-Mis18 $\alpha$ cells transfected with Aurora B wild-type (Aurora B WT) or K160A kinase dead mutant (Aurora B KD) were used for IP with anti-p-Ser antibody. (G) Aurora B kinase consensus sequences in mouse and human Mis18 $\alpha$. (H) Dot blot analysis for a phosphorylation-specific antibody of Mis18 $\alpha$ on Ser36 (p-Mis18 $\alpha$ ) by comparing non-phospho peptide with phospho-peptide at indicated concentrations. (I) Extracts from 293T cells transfected with Flag-Mis18 $\alpha$ were treated with $\lambda$-phosphatase and used for immunoblotting with anti-p-Mis $18 \alpha$ antibody. (J) 293T cells were transfected with Flag-Mis18 $\alpha$ WT, Flag-Mis18 $\alpha$ SA and synchronized by nocodazole treatment. Cell extracts were used for immunoblotting with anti-p-Mis18 $\alpha$ antibody. (K) Recombinant His-H3 or His-Mis18 $\alpha$ were incubated with purified Aurora B kinase in the presence of ATP for $30 \mathrm{~min}$ at $30^{\circ} \mathrm{C}$ for in vitro kinase assay. Phosphorylation of Mis $18 \alpha$ was detected using anti-p-Mis18 $\alpha$. (L) 293T cells expressing Flag-Mis18 $\alpha$ WT were synchronized by nocodazole treatment. After releasing, cells were harvested at indicated time points and applied for immunoblotting. (M) 293T cells expressing Flag-Mis18 $\alpha$ WT were released for $6 \mathrm{~h}$ from nocodazole-mediated synchronization with or without MG132 treatment to block APC activity. (N) HeLa/Flag-Mis18 $\alpha$ cells were released for $6 \mathrm{~h}$ from nocodazole-mediated synchronization as in M and subject to IP analysis with anti-Aurora B antibody. (O) HeLa/ Flag-Mis $18 \alpha$ cells expressing Flag-Mis $18 \alpha$ were treated with Aurora B kinase inhibitor, Hesperadin and the phosphorylation of Mis18 $\alpha$ was evaluated by using anti-p-Mis $18 \alpha$ antibody under nocodazole treatment.

then released for $30 \mathrm{~min}$. With the representative data for prometaphase cells (Figure 3E, left) and G1 phase cells (Figure 3E, right), knockdown of Mis $18 \alpha$ in HeLa cells diminished CENP-A dots dramatically on day 7; only $13 \%$ of cells showed positive signal for CENP-A dots compared with the control shRNA-infected cells (Figure $3 \mathrm{~F})$. However, reconstitution of Mis $18 \alpha$ WT and Mis $18 \alpha$ SA recovered CENP-A dots approximately up to $80 \%$ in centromere. Taken together, we could exclude the effect of Mis $18 \alpha$ phosphorylation on newly synthesized CENP-A loading into centromere.

\section{Mis18 $\alpha$ phosphorylation enhances PLK1 kinetochore recruitment}

Aurora B kinase functions to regulate kinetochoremicrotubule attachment during prometaphase and PLK1 is another key regulator for this function with Aurora B kinase $[14,20]$. To achieve accurate microtubule binding to kinetochore, Aurora B kinase and PLK1 phosphorylate each substrate at a balanced level for microtubule dynamics. If either kinase is abnormally activated, the cells divide with abnormal microtubule binding inducing misaligned chromosomes $[14,20]$. We questioned whether the mitotic defects shown in Mis18 $\alpha$ SA-reconstituted cells are caused by dysregulation of PLK1 at the kinetochore during prometaphase. Based on this hypothesis, we examined PLK1 recruitment to the kinetochore during prometaphase compared to Mis $18 \alpha$-depleted HeLa cells. Mis18 $\alpha$ knockdown was validated by the disappearance of CENP-A dots, while anti-centromere antibody (ACA) recognized centromere regions in the chromosomes (Figure 4A). In control cells, PLK1 dots were clearly stained consistently with centromere markers, whereas the intensity of PLK1 dots was significantly reduced at the kinetochore in Mis $18 \alpha$-knockdown cells (Figure 4B), indicating that Mis $18 \alpha$ is necessary for the proper recruitment of PLK1 to the kinetochore. Interestingly, introduction of Mis $18 \alpha$ WT recovered the intensity of PLK1 dots; however, introduction of Mis18 $\alpha$ SA was not sufficient to substitute for Mis $18 \alpha$ WT (Figure 4C). The evaluation of the ratio of PLK1 dots to ACA centromere maker confirmed that Mis $18 \alpha$ phosphorylation is necessary for the proper recruitment of PLK1 to kinetochore (Figure 4D). Since Aurora B is also responsible for PLK1 activation at kinetochore through Thr210 phosphorylation [14], we next checked whether Mis18 $\alpha$ phosphorylation is also involved in it. Interestingly, Thr210 phosphorylation of PLK1 was also decreased in Mis18 $\alpha$ SA-reconstituted cells, indicating that Mis $18 \alpha$ phosphorylation is essential for the function of PLK1 at kinetochore (Figure 4E). Moreover, Mis18 $\alpha$ SD, a phospho-mimic form, recovered and further maintained PLK1 recruitment even during the metaphase when PLK1 starts to leave kinetochore [20] (Figure 4F). The level of PLK1 in Mis18 $\alpha$ WT- or Mis18 $\alpha$ SA-reconstituted cells was comparable, indicating that reduced kinetochore recruitment of PLK1 in Mis18 $\alpha$ SA-reconstituted cells is not related to its protein level (Figure 4G). Taken together, these results indicate that Mis $18 \alpha$ phosphorylation by Aurora B kinase is necessary for the PLK1 kinetochore localization at early mitosis.

\section{PLK1 recognizes Mis18 $\alpha$ phosphorylation through its Polo Box Domain (PBD)}

To examine whether Mis18 $\alpha$-PLK1 binding is important for PLK1 kinetochore recruitment and whether Mis $18 \alpha$ phosphorylation mediates the binding, we performed co-immunoprecipitation assay. Interestingly, PLK1 bound with Mis18 $\alpha$ WT, but the binding with Mis $18 \alpha$ SA decreased significantly (Figure 5A). In addition, the binding between PLK1 and Mis18 $\alpha$ SD phospho-mimic mutant increased more than Mis18 $\alpha$ WT (Figure 5B). In vitro binding assay was also performed to confirm if Mis $18 \alpha$ phosphorylation is necessary for its interaction with PLK1. Mis18 $\alpha$ interacted with PLK1 only in the presence of ATP and Aurora B kinase, whereas Mis18 $\alpha$ SA did not bind with PLK1 even in the presence of ATP and Aurora B kinase (Figure 5C). In a separate assay, phosphorylation mimic mutant form of Mis18 $\alpha$ (Mis18 $\alpha$ SD) interacted with PLK1 without addition of Aurora B kinase (Supplementary Figure 5). Furthermore, knockdown of Aurora B kinase diminished the binding between PLK1 and Mis18 $\alpha$ (Figure 5D), and overexpression of Aurora B kinase increased their binding (Figure 5E), revealing Aurora B kinase dependent binding of PLK1. Nocodazole treatment increased the binding between PLK1 and Mis18 $\alpha$ (Figure 5F). Thus, 
A

HeLa stable cells (+pLKO-shMis 18 $\alpha$ )

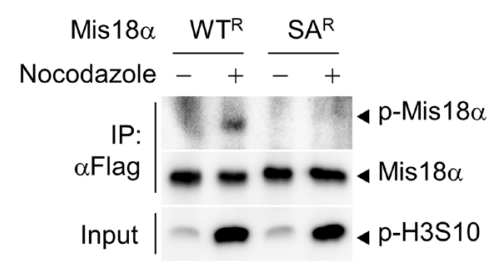

B

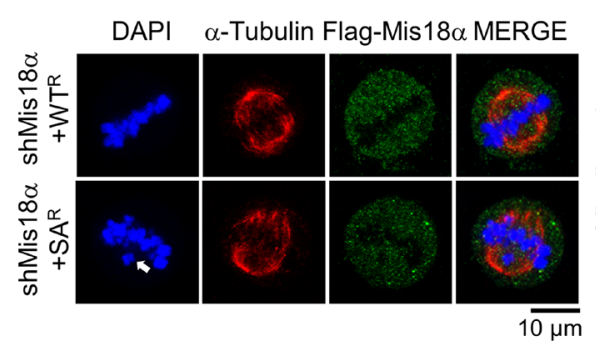

C
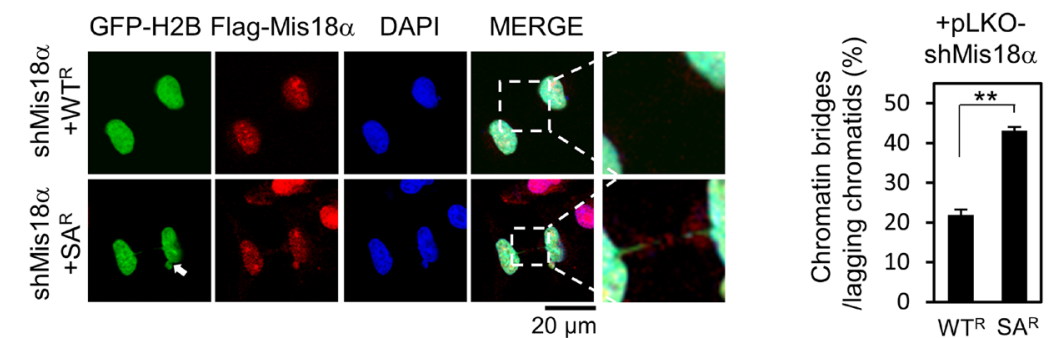

$\overline{10 \mu \mathrm{m}}$

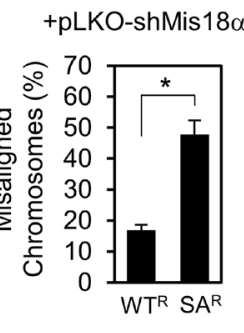

+ pLKO-

shMis $18 \alpha$

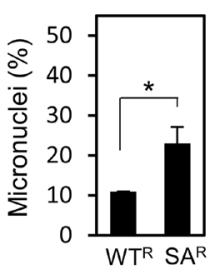

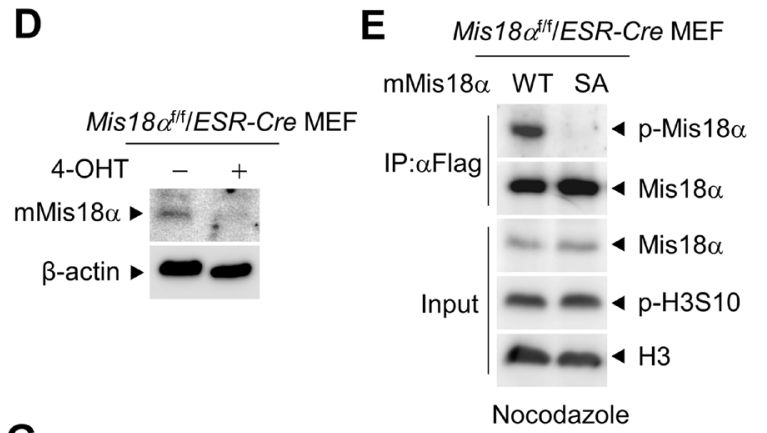

\section{$\mathbf{F}$}

G
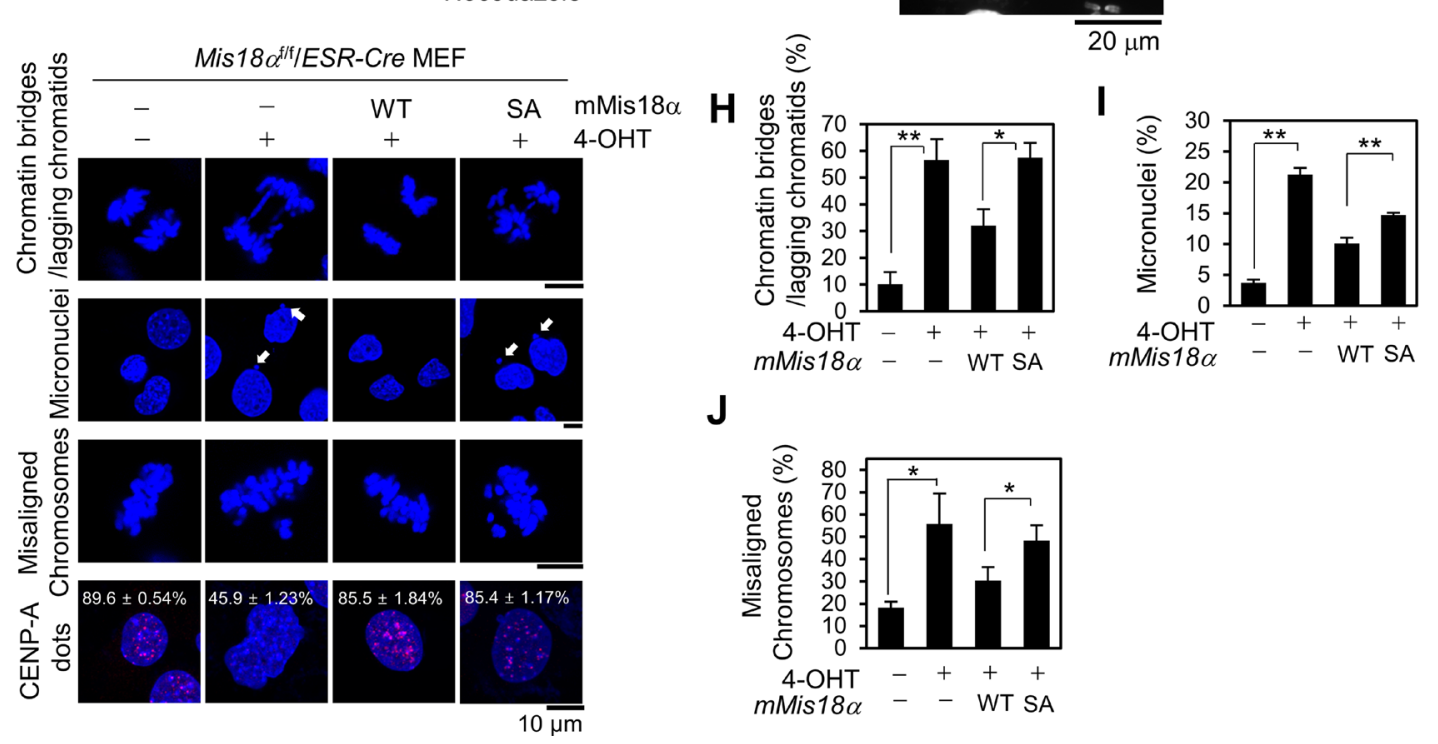

Figure 2: Mis18 $\alpha$ phosphorylation is necessary for faithful mitotic division. (A) Immunoblotting for Mis $18 \alpha$ phosphorylation in nocodazole-synchronized HeLa/Flag-Mis18 $\alpha$ stable cells. WT ${ }^{\mathrm{R}}$ or SA ${ }^{\mathrm{R}}$ represents shRNA-resistant form of Mis18 $\alpha$ proteins. (B) Images of misaligned chromosomes in HeLa/Flag-Mis18 $\alpha$ stable cells (left) and the number of cells showing misaligned chromosomes presented in percentage (right). $P$ value is calculated by $t$-test $(" p<0.05)$. (C) Images of the chromatin bridges or lagging chromatids and micronuclei in $\mathrm{HeLa} /$ Flag-Mis $18 \alpha$ stable cells (left) and the number of cells showing the chromatin bridges or lagging chromatids presented in percentage (right). $P$ value is calculated by $t$-test $\left({ }^{*} p<0.05\right.$ ). (D) Mis $18 \alpha^{f f f} E S R$-Cre MEFs were treated with 4-hydroxy-tamoxifen (4-OHT) for four days and the depletion of endogenous mMis18 $\alpha$ was validated by immunoblot using anti-mMis18 $\alpha$ antibody. (E) The phosphorylation

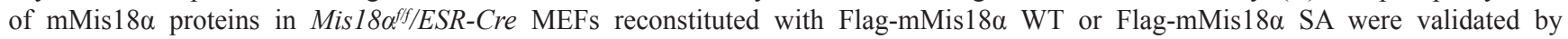


immunoblotting with anti-p-Mis18 $\alpha$ antibody. (F) Reconstituted Mis $18 \alpha^{\text {ff }} / E S R$-Cre MEFs were analyzed by chromosome spreading assay. Histogram in right side shows the percentage of cells containing chromosome number over $4 \mathrm{~N}$ for each genotype. The number of chromosome spreads is 120 each and chromosomes were counted using Image $\mathrm{J}$ software by identifying the centromeres, the brighter part than the rest of the chromosome. $P$ value is calculated by $t$-test $\left({ }^{* * *} p<0.001\right)$. (G) The chromatin bridges or lagging chromatids, micronuclei and misaligned chromosomes were analyzed in reconstituted Mis $18 \alpha^{f f f} / E S R$-Cre MEFs in parallel with CENP-A dots. (H-J) The percentage of cells showing the chromatin bridges or lagging chromatids $(\mathrm{H})$, micronuclei $(\mathrm{I})$, and misaligned chromosomes (J) was calculated from the images in G. $P$ value is calculated by $t$-test $\left({ }^{*} p<0.05,{ }^{* *} p<0.01\right)$.

phosphorylation of Mis $18 \alpha$ enhances its binding with PLK1.

PLK1 localization is enhanced by phospho-binding domain, called Polo Box Domain (PBD) [21, 22]. PLK1 recognizes substrates through its $\mathrm{PBD}$, and substrate binding through PBD leads to further activation of PLK1. Therefore, we checked whether the PBD of PLK1 is involved in the binding with the phosphorylated Mis 18a. Since two sites (His538 and Lys540) in PBD are important for the binding of PLK1 to other substrates [23], we generated PLK1 AA mutant by substituting each amino acid to alanine. Interestingly, the binding of PLK1 AA with Mis $18 \alpha$ decreased considerably compared to PLK1 WT (Figure 5G). PLK1 AA mutant also exhibited decreased binding to Aurora B kinase (Figure $5 \mathrm{H}$ ). To gain more insight into PLK1 and Mis18 $\alpha$ interaction, we performed bimolecular fluorescence complementation assay [24]. The N-terminal half and the $\mathrm{C}$-terminal half of VENUS were fused with Mis18 $\alpha$ and PLK1, respectively. When the two proteins were co-expressed, Mis $18 \alpha /$ PLK1 interaction-mediated complementation of VENUS showed green fluorescence signals. However, Mis18 $\alpha$ SA or PLK1 AA mutant failed to show any fluorescence signal (Figure 5I). Taken together, PLK1 binds to phosphorylated Mis $18 \alpha$ through its PBD and the binding enhances PLK1 recruitment to the kinetochore at prometaphase (Figure 6).

\section{DISCUSSION}

Mis $18 \alpha$ is a component of Mis 18 complex that is crucial for centromere deposition of newly synthesized CENP-A at early G1 phase of cell cycle $[4,6]$. In this study, we identified a distinct role of Mis18 $\alpha$ at mitosis, independent of its known function in CENP-A deposition at early G1 phase. The mitotic function of Mis $18 \alpha$ is accompanied by Aurora B kinase-mediated phosphorylation on a conserved serine residue (Ser36 in human and Ser13 in mouse) during mitosis and this phosphorylation enhances the interaction between Mis $18 \alpha$ and PLK1 resulting in the increased kinetochore recruitment of PLK1. The Mis18 $\alpha$ phosphorylation we have shown here is distinct from previously identified phosphorylations of Mis 18 proteins by CDK $1 / 2$ and PLK1, which mainly regulate the function for CENP-A deposition. First, it occurs during mitosis specifically by Aurora B kinase, and the association with Mis18BP1 is not necessary for the phosphorylation. Second and most importantly, the phosphorylation is independent of CENP-A loading. Phosphorylation-defective mutation of
Mis $18 \alpha$ does not affect either timely scheduled centromere localization of Mis18 complex during cell cycle progression or its function as a priming factor for CENP-A deposition. In detail, the replacement of endogenous Mis $18 \alpha$ with phosphorylation-defective Mis $18 \alpha$ in two cell types, HeLa and MEFs, did not induce CENP-A loss, whereas Mis $18 \alpha$ depletion without Mis $18 \alpha$ reconstitution clearly caused CENP-A loss.

It has been previously shown that the depletion of Mis $18 \alpha$ causes mitotic defects such as misaligned chromosomes and chromosomal bridges [3], which finally leads to cell death resulting in developmental failure of embryos and skin stratification in mice $[3,5]$. Interestingly, the expression of phosphorylation-defective mutant form of Mis18 $\alpha$ in Mis $18 \alpha$-depleted cells can rescue severe lethal phenotype of Mis $18 \alpha$ depletion; however, still exhibits mitotic defects, indicating that although the phosphorylation is not critical for CENP-A deposition, it contributes to the faithful chromosome alignment and segregation. Indeed, Aurora B kinase and PLK1 are essential kinases for accurate KT-MT attachment by interdependent regulation and the loss of Aurora B kinase activity is known to cause abnormally stable KTMT attachment, as the cooperation with PLK1 to balance $\mathrm{KT}$ and MT tension is collapsed [25-28]. This results in abnormally increased inter-kinetochore distance [29]. Aurora B kinase destabilizes KT-MT attachment, whereas PLK1 stabilizes it by phosphorylating BubR1 to disturb Aurora B kinase activity [25]. However, PLK1 activates Aurora B kinase by phosphorylating Survivin, which is a member of chromosomal passenger complex and Aurora B kinase activates PLK1 by direct phosphorylation [14, 15]. Due to this complicated cooperation, cells may have multilayers of self-checking system for accurate KT-MT attachment and Mis $18 \alpha$ phosphorylation by Aurora B kinase would have a role of enhancing PLK1 recruitment.

The most striking feature of PLK1 regulation is PBD-dependent interaction with its substrates resulting in the kinetochore localization. There are many studies on the proteins that recruit PLK1 to the kinetochore by investigating their interaction with PBD. The PBIP1 recruits PLK1 by PBD-mediated binding at early mitosis and PLK1 phosphorylates PBIP1 for selfprimed enrichment [30]. Interestingly, PLK1-mediated phosphorylation of PBIP1 on another site induces its degradation and releases PLK1 for the interaction with other recruiting factors. Indeed, PLK1 kinetochore signals remain even when the PBIP1 is depleted [31], indicating multilayered regulation of PLK1 kinetochore 
A

B
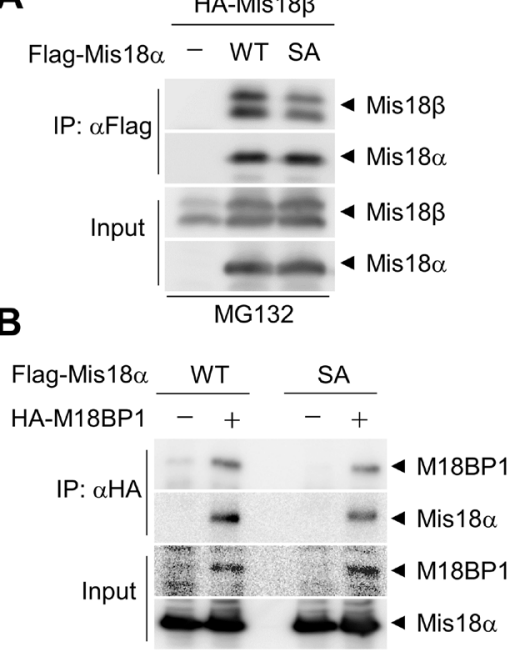

D

HeLa cells stably expressing Flag-Mis $18 \alpha$

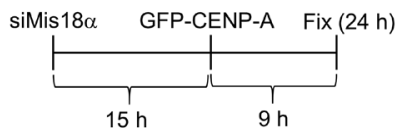

E

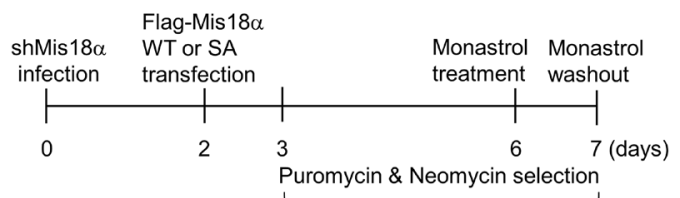

DAPI CENP-A Aurora B MERGE

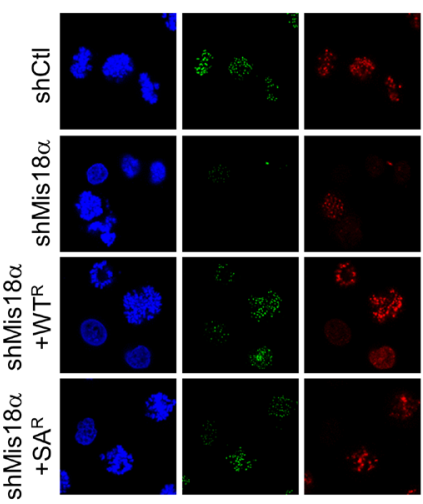

Prometaphase

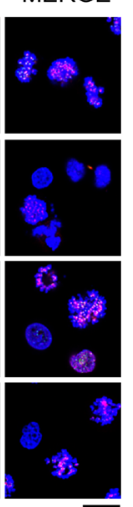

$20 \overline{\mu m}$
C
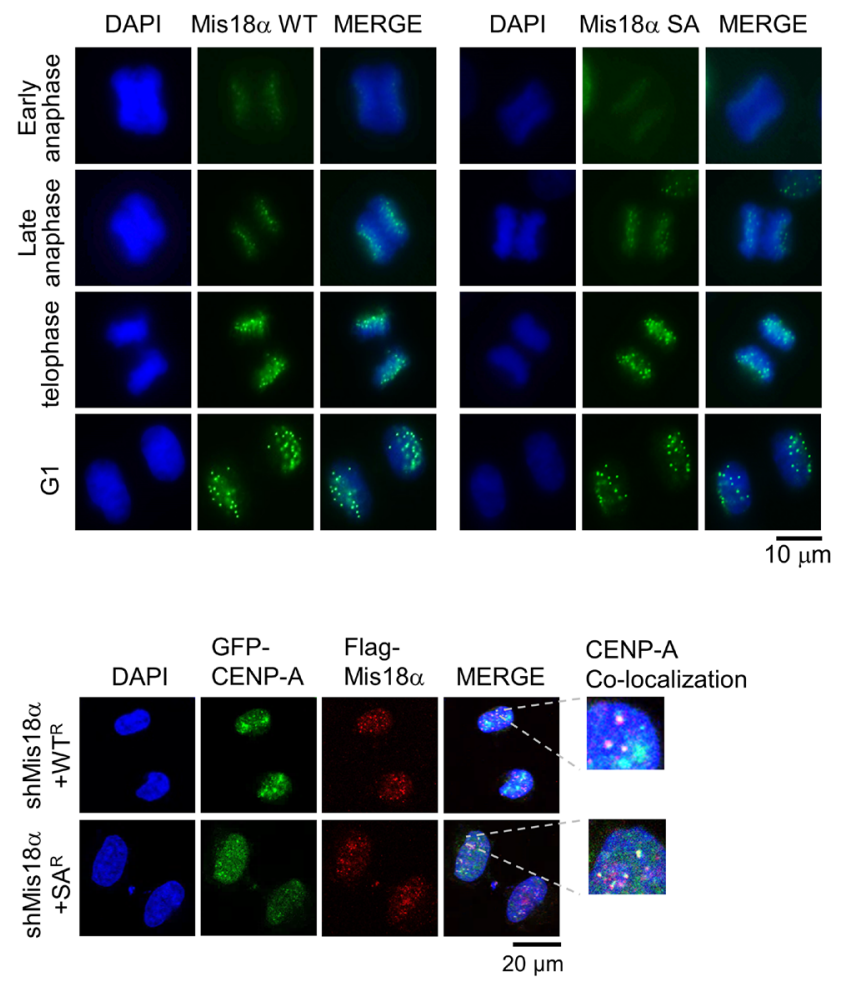

$\mathbf{F}$

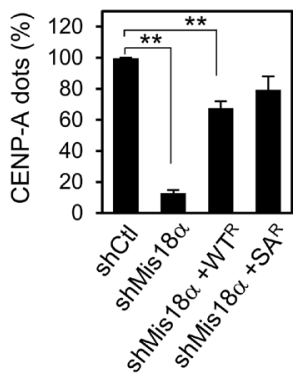

Figure 3: Mis18 $\alpha$ phosphorylation is not required for CENP-A loading. (A) HA-Mis18 $\beta$ with either Flag-Mis18 $\alpha$ WT or Flag-Mis $18 \alpha$ SA were transfected into 293T cells and the extracts were applied for co-IP assay. (B) The binding between Flag-Mis18 $\alpha$ and HA-M18BP1 was analyzed as in A. (C) HeLa/Flag-Mis18 $\alpha$ stable cells were synchronized by double-thymidine block and released into indicated phase. The cells were stained with anti-Flag antibody. The green dots indicate centromeric localization of Mis $18 \alpha$. Confocal image with 1,000x magnification. (D) Analysis scheme for the centromere recruitment of newly synthesized CENP-A (left). HeLa cells stably expressing siRNA-resistant form of Mis $18 \alpha$ WT $\left(\mathrm{WT}^{\mathrm{R}}\right)$ or SA $\left(\mathrm{SA}^{\mathrm{R}}\right)$ were transfected sequentially with siRNA against Mis $18 \alpha$ and with GFP-CENP-A (mimic newly synthesized CENP-A) as indicated in the scheme. Immunocytochemistry for Mis18 $\alpha$ with anti-Flag antibody and GFP-CENP-A (right). (E) Scheme for the centromeric recruitment of CENP-A under prolonged Mis18 $\alpha$ knockdown (upper). Knockdown of endogenous Mis18 $\alpha$ was achieved by infecting lentivirus that is expressing shRNA against Mis $18 \alpha$. Lower left panel shows CENP-A dots in prometaphase cells and lower right panel represents G1 phase cells. (F) The number of CENP-A dot positive cells from E were calculated and expressed as a percentage of total cells. $P$ value is calculated by $t$-test $\left.{ }^{* * *} p<0.01\right)$. 
A

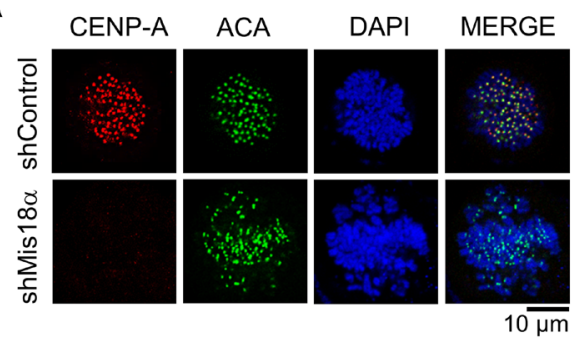

C

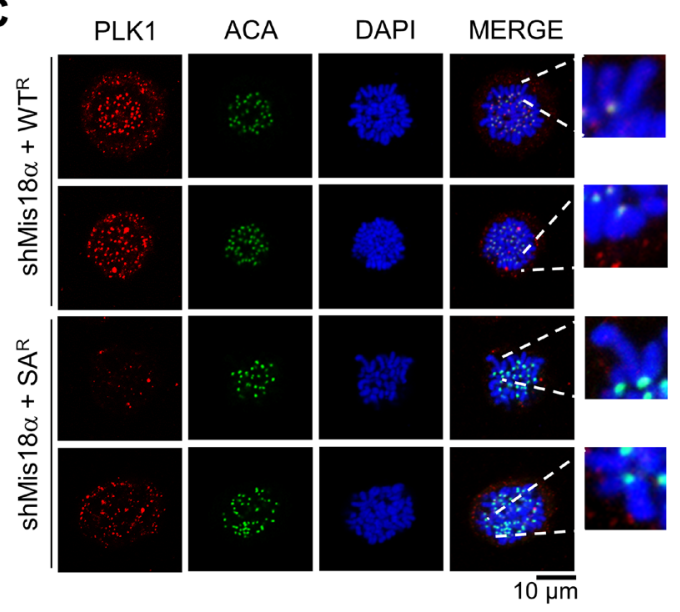

D

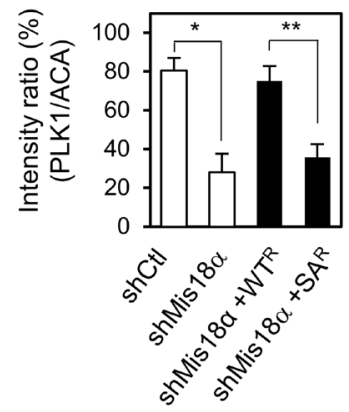

$\mathbf{F}$

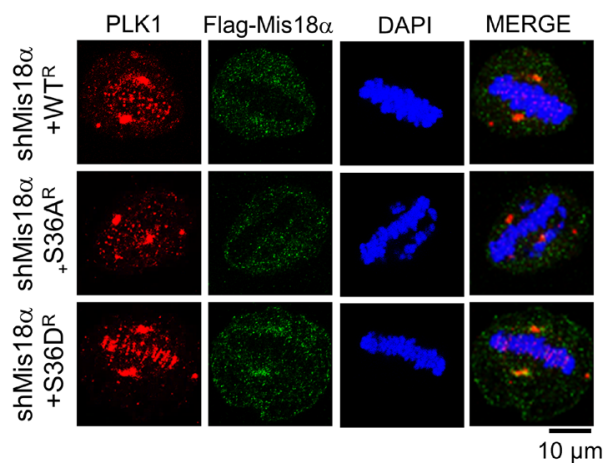

B

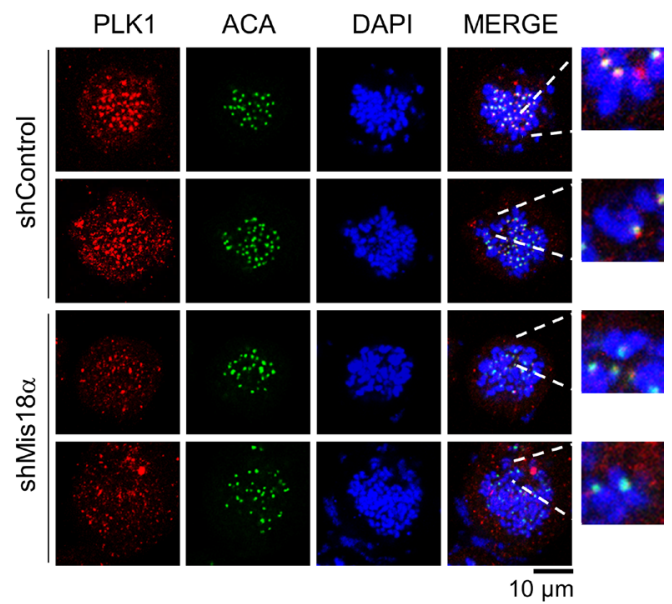

E

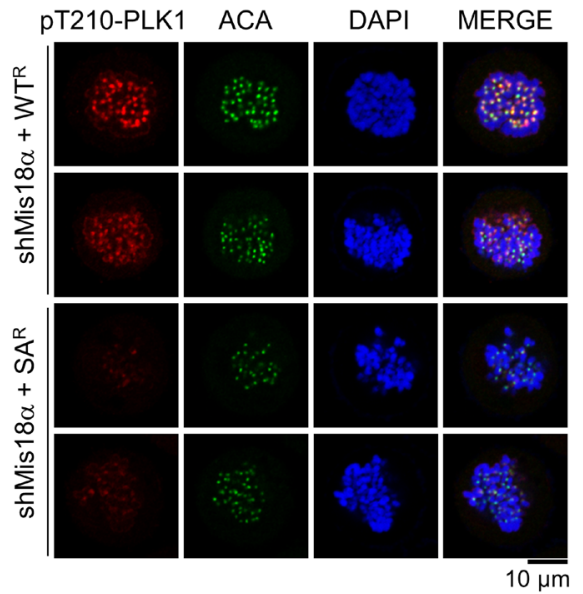

G

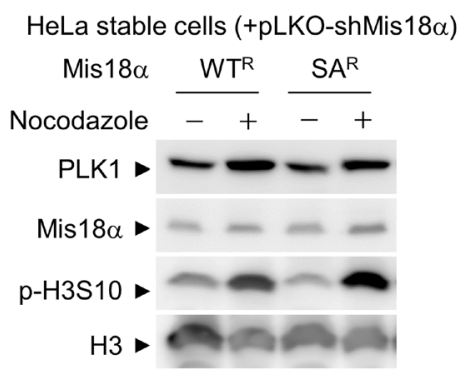

Figure 4: Mis18a phosphorylation enhances PLK1 kinetochore recruitment. (A) HeLa cells were infected with lentivirus expressing either control shRNA (shControl) or shRNA against Mis $18 \alpha$ (shMis18 $\alpha$ ). Cells were fixed at prometaphase by releasing for 30 min after monastrol treatment and stained with anti-ACA (centromere marker) or anti-CENP-A antibody. Confocal image with 1,000× magnification. (B) Cells prepared as in A were co-stained with anti-PLK1 and anti-ACA antibodies. (C) HeLa cells stably expressing shRNA-resistant form of Mis $18 \alpha\left(\right.$ WT $^{\mathrm{R}}$ and $\mathrm{SA}^{\mathrm{R}}$ ) were infected with lentivirus expressing shMis18 $\alpha$. Cells were co-stained with anti-PLK1 and anti-ACA antibodies at prometaphase. (D) The number of cells showing high intensity of PLK1 staining, ACA signal as a control (PLK1/ACA), from B and C was presented in percentage. $P$ value is calculated by $t$-test $\left({ }^{*} p<0.05,{ }^{* *} p<0.01\right)$. (E) pThr210-PLK1 was co-stained with ACA in the same cells as C. (F) HeLa cells stably expressing shRNA-resistant form of Mis $18 \alpha\left(\mathrm{WT}^{\mathrm{R}}, \mathrm{SA}^{\mathrm{R}}\right.$ and $\left.\mathrm{SD}^{\mathrm{R}}\right)$ were co-stained with anti-PLK1 and anti-Flag Mis18 $\alpha$ antibody at metaphase. (G) Immunoblot for PLK1 level in reconstituted HeLa stable cell lines. 
A

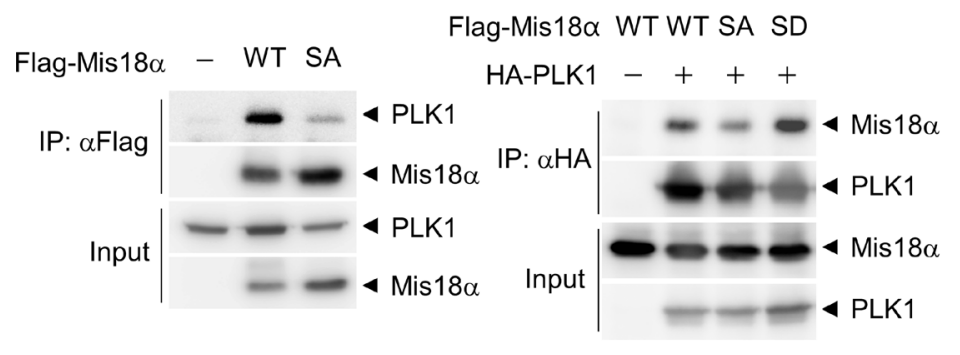

D

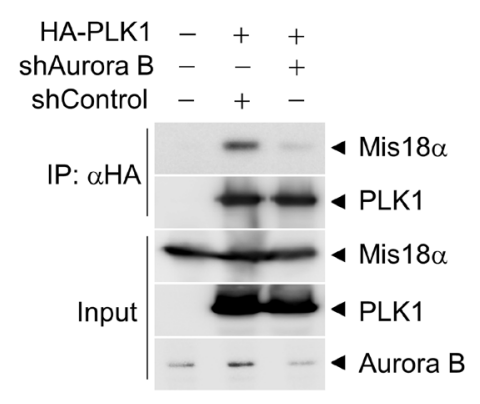

E

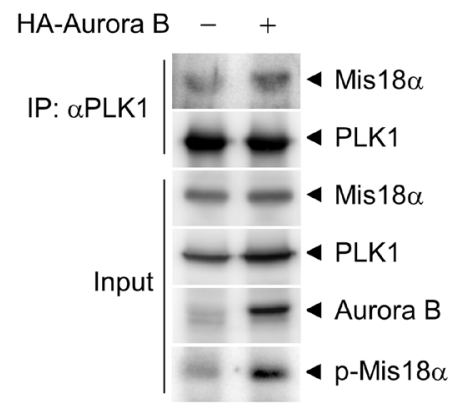

C

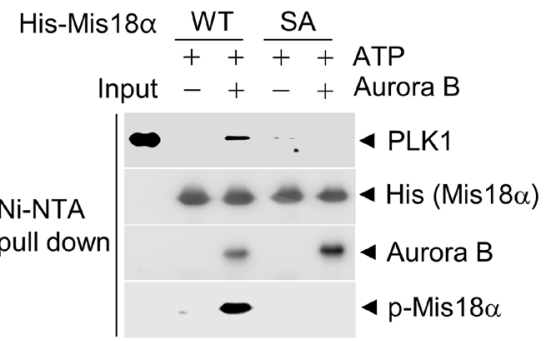

F

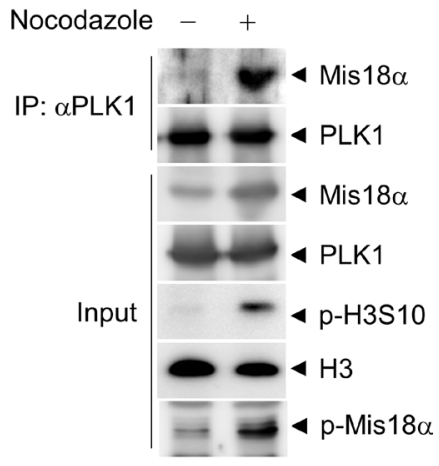

G

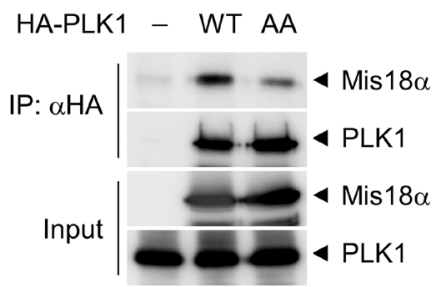

H

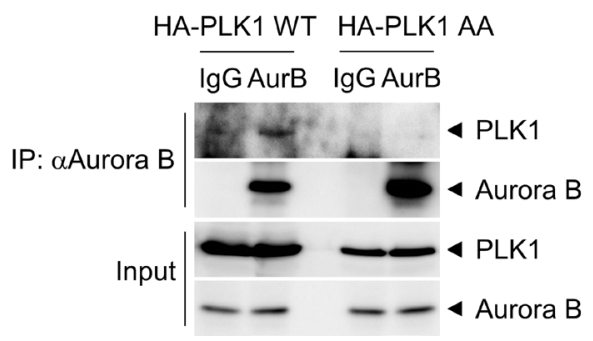

I

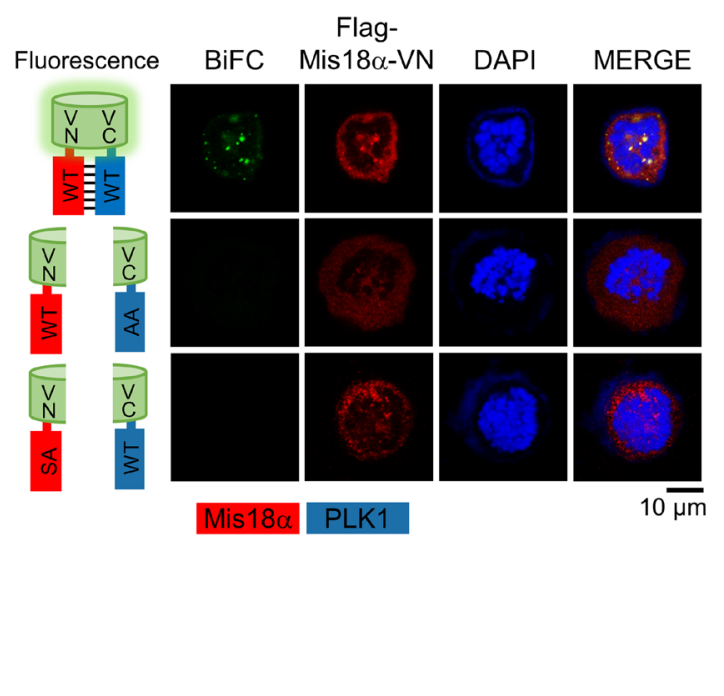

Figure 5: PLK1 recognizes Mis18a phosphorylation through its PBD. (A) Flag-Mis18 $\alpha$ and HA-PLK1 constructs were transfected in $293 \mathrm{~T}$ cells and cell extracts were applied for IP analysis by using anti-Flag antibody. (B) Flag-Mis18 $\alpha$ and HA-PLK1 constructs were transfected in 293T cells and cell extracts were applied for IP analysis by using anti-HA antibody. (C) HA-PLK1 was synthesized in vitro by using a coupled Transcription/Translation system and incubated with recombinant His-Mis $18 \alpha$ in the presence of Aurora B kinase for in vitro binding assay. The sample was subjected to immunoblotting with anti-HA antibody. (D) 293T cells were transfected as in A in the presence or absence of shRNA against Aurora B kinase. IP was performed by using anti-HA antibody. (E-F) FlagMis $18 \alpha$ constructs were transfected in 293T cells in the presence of Aurora B kinase overexpression (E) or nocodazole treatment (F), and IP was performed by using anti-PLK1 antibody. (G) Flag-Mis18 $\alpha$ was transfected into 293T cells together with either wild-type PLK1 (HAPLK1 WT) or PBD-mutant form of PLK1 (HA-PLK1 AA). IP was performed using anti-HA antibody. (H) 293T cell extracts expressing either HA-PLK1 WT or HA-PLK1 AA were applied for IP analysis using anti-Aurora B kinase antibody. (I) Bimolecular fluorescence complementation assay. Flag-Mis $18 \alpha-\mathrm{VN}$ constructs and HA-PLK1-VC constructs were transfected into HeLa cells and the fluorescence images were detected under confocal microscope (green fluorescence for BiFC and red fluorescence for Mis $18 \alpha$ ). Confocal image with $1,000 \times$ magnification. 
recruitment. In addition, INCENP and RSF1 are known to function in PLK1 kinetochore recruitment. Knockdown of INCENP, which is a member of passenger complex with Aurora B kinase, resulted in the loss of PLK1 kinetochore recruitment [32] providing the evidence of Aurora B complex's involvement in PLK1 recruitment. RSF1 partially regulates PLK1 kinetochore recruitment and dual knockdown of RSF1 and INCENP further reduces PLK1 kinetochore recruitment [33]. In our study, Mis $18 \alpha$ SA reconstitution did not completely inhibit PLK1 recruitment to the kinetochore. Moreover, there was no obvious mitotic delay. Therefore, we speculate that the mitotic defects observed from Mis18 $\alpha$ SA-reconstituted cells would be the accumulated defects from prolonged dysregulation of PLK1.

In summary, we have identified mitosis-specific phosphorylation of Mis $18 \alpha$ by Aurora B kinase, which is not essential for the previously known function of Mis 18 complex in CENP-A deposition to centromere. Instead, the phosphorylation of Mis $18 \alpha$ contributes the recruitment of PLK1 to the kinetochore, which requires PBD-mediated binding of phosphorylated Mis $18 \alpha$ at prometaphase. Since the phosphorylation-defective mutation on Mis $18 \alpha$ causes mitotic problems, Mis $18 \alpha$ plays a critical role in mitosis in addition to its well-known function in CENP-A deposition at G1 phase. Our finding opens up a possibility that Mis $18 \alpha$ may play a diverse role in a wide range of cell cycle regulation.

\section{MATERIALS AND METHODS}

\section{Cell culture, generation of stable cells and transfection}

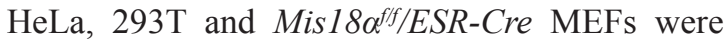
cultured in $37^{\circ} \mathrm{C}$ humidified $\mathrm{CO}_{2}$ incubator with DMEM containing $10 \%$ FBS and antibiotics. All cell lines were regularly tested for mycoplasma contamination. For the generation of Mis18 $\alpha$-stably reconstituted HeLa cell lines, cells transfected with shRNA-resistant Flag-Mis $18 \alpha$ were selected with neomycin for two weeks. The cells were then infected with shMis $18 \alpha$ expressing lentivirus (pLKO-shMis 18 $\alpha$ ) followed by selection with puromycin. Lentivirus was generated by transfecting lentiviral shRNA and packaging plasmids (psPAX2 and pMD2.G) into 293T cells. The culture supernatant were collected two days later and concentrated by Lenti-concentrator (Takara Bio, USA). The targeting sequences of shRNA are as follows; human Mis18 $\alpha$, 5'-CAGAAGCTATCCAAACGTG-3'; human M18BP1, 5'-GGATATCCAAATTATCTCA-3'. The targeting sequence of siRNA for human Mis $18 \alpha$ is as follows; 5'-CAGAAGCUAUCCAAACGUGUU-3'. For the generation of Mis $18 \alpha$-reconstituted Mis $18 \alpha^{f / f} /$ ESR-Cre MEF cell lines, cells were infected with FlagmMis18 $\alpha$ expressing lentivirus (pLJM1-Flag-Mis18 $\alpha$ ) and selected with puromycin for two weeks. For the depletion of endogenous mMis18 $\alpha$, 4-hydroxy-tamoxifen (200 nM) was added for 4 days.

\section{Cell synchronization}

To arrest cells at G1/S, the cells were incubated with DMEM media containing $4 \mathrm{mM}$ thymidine (Sigma, St Louis, MO) for $16 \mathrm{~h}$ and were washed with PBS twice. After $9 \mathrm{~h}$ of release, cells were incubated with thymidine containing media again for $15 \mathrm{~h}$, and were released and harvested at indicated time points. To arrest cells at metaphase, the cells were incubated with DMEM media containing $0.4 \mu \mathrm{g}$ nocodazole (Sigma, St Louis, MO) for $15 \mathrm{~h}$. For metaphase-aligned chromosomes, MG132 (Sigma, St Louis, MO) was added while cells were released from $100 \mathrm{nM}$ monastrol incubation for $15 \mathrm{~h}$.

\section{Bimolecular fluorescence complementation assay}

The N-terminal half of Venus fluorescent protein with Flag-tag was fused with Mis $18 \alpha$ and the C-terminal half of Venus with HA-tag was fused with PLK1.

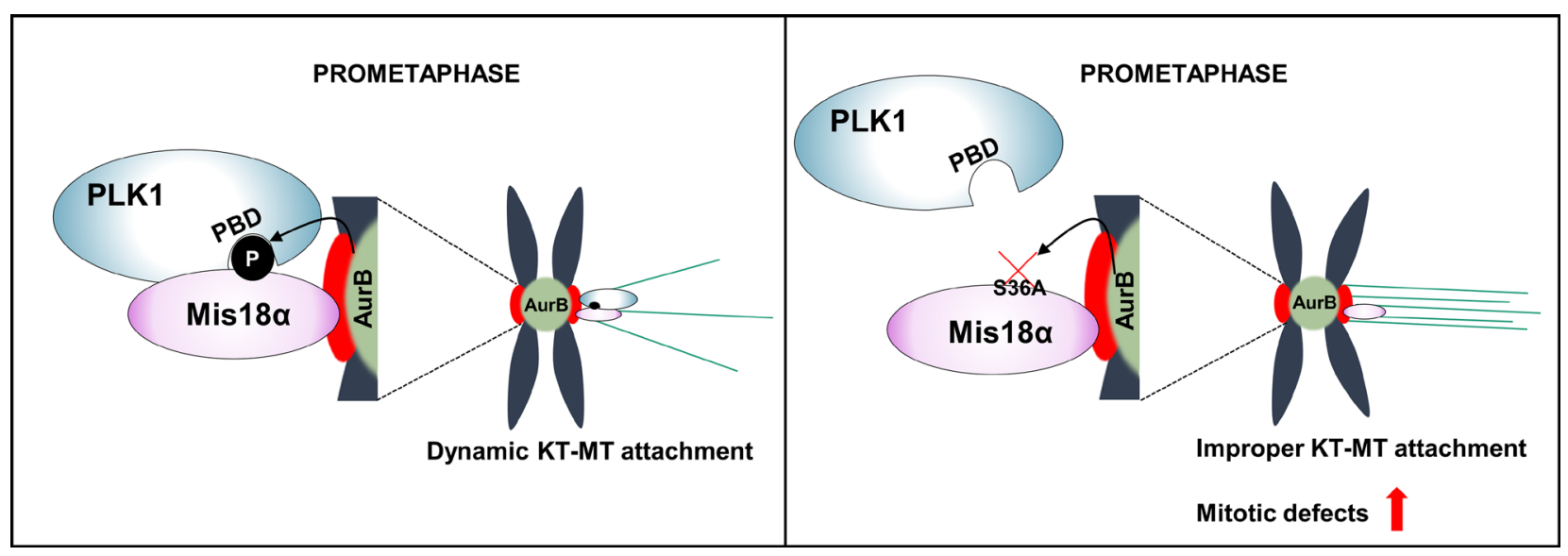

Figure 6: Mis18 $\alpha$ function during mitosis by enhancing PLK1 kinetochore recruitment. A schematic model showing how Mis $18 \alpha$ phosphorylation enhances PLK1 recruitment at the kinetochore. 
Phosphorylation-defective mutant of Mis $18 \alpha$ and PBD mutant of PLK1 were fused with Venus in the same way, respectively. These constructs were transfected into HeLa cells in combination. The cells were synchronized by monastrol treatment and then released to obtain prometaphase population. The expression of each construct was validated by immunostaining with anti-Flag or anti-HA antibodies. The complemented Venus protein was detected by green fluorescence signal using a confocal microscope (Carl Zeiss, Germany).

\section{Immunoprecipitation}

The whole cell lysates were prepared using lysis buffer (50 mM Tris- $\mathrm{HCl}$ pH 8.0 containing $200 \mathrm{mM} \mathrm{NaCl}$, $0.5 \%$ NP-40, and freshly added protease and phosphatase inhibitors). Cell lysates were briefly sonicated to shear the chromatin structure. For immunoprecipitation, $1 \mathrm{mg}$ of lysates were incubated sequentially with primary antibody for $4 \mathrm{~h}$ followed by protein $\mathrm{A} / \mathrm{G}$ coated beads for $1 \mathrm{~h}$ at $4^{\circ} \mathrm{C}$.

\section{Immunoblot}

For immunoblot, normalized cell lysates or immunoprecipitation samples were separated on SDSPAGE gels and transferred on nitrocellulose membrane (GE Healthcare, Little Chalfont, UK). The blots were probed with the following primary antibodies; anti-Flag (Sigma, St Louis, MO), anti-HA (Covance, Princeton, NJ), anti-H3 (Cell signaling, Danvers, MA), anti-pH3S10 (Abcam, Cambridge, UK), anti-Aurora B (Abcam, Cambridge, UK), anti-PLK1 (Santa Cruz, Dallas, TX), anti-ACA (Antibodies Incorporated, Davis, CA), antiCENP-A (Cell Signaling, Danvers, MA), anti-phospho serine (Sigma, St Louis, MO). Phosphorylation-specific antibody for Mis $18 \alpha$ was generated by injecting synthetic phospho-peptide to rabbits and purified using phosphopeptide affinity chromatography (AbClone, Seoul, South Korea). Peptide sequence used for injection is as follows; 5'-CESPLLEKRL(pS)EDSSR-3'.

\section{Immunocytochemistry}

For immunocytochemistry, the cells were cultured on poly-L-Lysine coated coverslip or chamber slide and were fixed with $2 \%$ formaldehyde for $15 \mathrm{~min}$ at $25^{\circ} \mathrm{C}$. Cells were then permeabilized with PBS containing $0.5 \%$ Triton $\mathrm{X}-100$ for $5 \mathrm{~min}$ followed by incubation with primary antibodies for overnight. After washing, secondary antibodies conjugated with Alexa Fluor 488 or 594 (Invitrogen, Carlsbad, CA) were applied in the dark for $1 \mathrm{~h}$ at $25^{\circ} \mathrm{C}$. DAPI was incubated for short time just before mounting and the slides were observed with confocal microscope (Carl Zeiss, Germany) at 1,000× magnification with immersion oil. The images were analyzed by Image $J$ software.

\section{In vitro binding assay}

HA-PLK1 was synthesized in vitro by using a coupled transcription and translation systems (Promega, USA). HA-PLK1 was incubated with recombinant GSTMis $18 \alpha$ for GST-pull down in binding buffer $(125 \mathrm{mM}$ $\mathrm{NaCl}, 20 \mathrm{mM}$ Tris-HCl pH 8.0, 10\% Glycerol, 0.1\% NP-40, $0.5 \mathrm{mM}$ DTT and protease inhibitors). The reaction was stopped by adding SDS sampling buffer and was subjected to SDS-PAGE. PLK1 was analyzed by immunoblotting with anti-HA antibody. Amount of GSTMis18 $\alpha$ was analyzed by anti-GST antibody.

\section{In vitro kinase assay}

Recombinant His-H3 and His-Mis $18 \alpha$ was incubated with purified Aurora B kinase (Eurofins, UK) for $30 \mathrm{~min}$ at $30^{\circ} \mathrm{C}$ in the kinase buffer $(50 \mathrm{mM}$ Tris- $\mathrm{HCl}, 10 \mathrm{mM}$ $\mathrm{MgCl}_{2}, 1 \mathrm{mM}$ EGTA, 2 mM DTT, 0.01\% Tween-20, 1 $\mathrm{mM}$ ATP, protease inhibitors and phosphatase inhibitors). The reaction was stopped by adding SDS sampling buffer and subjected to SDS-PAGE followed by immunoblotting with anti-p-H3S10 antibody and anti-p-Mis $18 \alpha$ antibody.

\section{In vitro kinase-binding assay}

Recombinant His-Mis $18 \alpha$ that is bound on Ni-NTAagarose was incubated with purified Aurora B kinase (Eurofins, UK) for $30 \mathrm{~min}$ at $30^{\circ} \mathrm{C}$ in the kinase buffer. The sample was washed with binding buffer and then incubated with in vitro synthesized HA-PLK1 in binding buffer for $2 \mathrm{~h}$ with rotating. After collecting the beads and washing with binding buffer, samples were boiled for 5 min with SDS sampling buffer and subjected to SDSPAGE. PLK1 was analyzed by immunoblotting with antiHA antibody.

\section{Chromosome spreading assay}

Mis $18 \alpha^{f f f} / E S R-C r e$ WT MEFs and Mis18 $\alpha^{f f f} / E S R$ Cre SA MEFs were incubated with colcemid (Sigma, USA) to a final concentration of $1 \mu \mathrm{g} / \mathrm{ml}$ for $4 \mathrm{~h}$ at $37^{\circ} \mathrm{C}$. After incubation, cells were trypsinized and harvested by centrifugation at 1,000 rpm for $4 \mathrm{~min}$. The pellet was resuspended with $75 \mathrm{mM} \mathrm{KCl}$ solution and incubated for $6 \mathrm{~min}$. After harvesting by centrifugation at 1,000 rpm for $4 \mathrm{~min}$, cells were fixed with methanol/glacial acetic acid (3:1) by dropping and mixing slowly. The fixed chromosomes were released as a single drop at a time onto the slide and were allowed to air-dry. The air-dried slide was covered by coverslip with DAPIcontaining mounting solution. The image was observed by confocal microscope (Carl Zeiss, Germany) at 1,000x magnification, and centromeres that are brighter than the rest of the chromosome were counted by Image $\mathrm{J}$ software. 


\section{Statistical analysis}

All experiments were performed independently at least three times. More than 100 cells were counted to evaluate mitotic defects in each experiment. For PLK1/ACA intensity ratio, an average number of 150 kinetochores was examined for each group. Values are expressed as mean \pm s.e.m. Significance was analyzed using two-tailed, unpaired $t$-test. $P<0.05$ was considered statistically significant.

\section{Abbreviations}

Microtubule (MT), Kinetochore (KT), Spindle Assembly Checkpoint (SAC), Polo Box Domain (PBD), Mitosis (M).

\section{Author contributions}

M.L., I.S.K., K.I.K. and S.H.B. designed the study; M.L. and I.S.K. performed the cell biology experiments; M.L. performed the immunocytochemistry analysis and in vitro assays; K.C.P. generated Mis $18 \alpha^{f f f} / E S R$-Cre MEFs; J.S.K. performed LC-MS/MS analysis; M.L., K.I.K. and S.H.B. wrote the manuscript; all authors contributed to data analysis.

\section{ACKNOWLEDGMENTS AND FUNDING}

This work was supported by Creative Research Initiatives Program (Research Center for Chromatin Dynamics, 2009-0081563) to S.H.B.; the Science Research Center program (Cellular Heterogeneity Research Center, NRF-2016R1A5A1011974) to K.I.K. from the National Research Foundation (NRF) grant funded by the Korea government (MSIP); Seoul Science fellowship and Brain Korea 21 fellowship to M.K.L. We thank K.E.P. for critical reading of our manuscript.

\section{CONFLICTS OF INTEREST}

None.

\section{REFERENCES}

1. Lesage B, Qian J, Bollen M. Spindle checkpoint silencing: PP1 tips the balance. Curr Biol. 2011; 21:R898-903.

2. Cleveland DW, Mao Y, Sullivan KF. Centromeres and kinetochores: from epigenetics to mitotic checkpoint signaling. Cell. 2003; 112:407-21.

3. Kim IS, Lee M, Park KC, Jeon Y, Park JH, Hwang EJ, Jeon TI, Ko S, Lee H, Baek SH, Kim KI. Roles of Mis $18 \alpha$ in epigenetic regulation of centromeric chromatin and CENP-A loading. Mol Cell. 2012; 46:260-73.

4. Fujita Y, Hayashi T, Kiyomitsu T, Toyoda Y, Kokubu A, Obuse C, Yanagida M. Priming of centromere for CENP-A recruitment by human hMis18 $\alpha$, hMis18 $\beta$, and M18BP1. Dev Cell. 2007; 12:17-30.

5. Park KC, Lee M, Jeon Y, Jeon R, Baek SH, Lee H, Kim KI. Skin-specific deletion of Mis18 $\alpha$ impedes proliferation and stratification of epidermal keratinocytes. J Invest Dermatol. 2017; 137:414-21.

6. Silva MC, Jansen LE. At the right place at the right time: novel CENP-A binding proteins shed light on centromere assembly. Chromosoma. 2009; 118:567-74.

7. Silva MC, Bodor DL, Stellfox ME, Martins NM, Hochegger H, Foltz DR, Jansen LE. Cdk activity couples epigenetic centromere inheritance to cell cycle progression. Dev Cell. $2012 ; 22: 52-63$.

8. McKinley KL, Cheeseman IM. Polo-like kinase 1 licenses CENP-A deposition at centromeres. Cell. 2014; 158:397-411.

9. Hirota T, Kunitoku N, Sasayama T, Marumoto T, Zhang D, Nitta M, Hatakeyama K, Saya H. Aurora-A and an interacting activator, the LIM protein ajuba, are required for mitotic commitment in human cells. Cell. 2003; 114:585-98.

10. Berdnik D, Knoblich JA. Drosophila Aurora-A is required for centrosome maturation and actin-dependent asymmetric protein localization during mitosis. Curr Biol. 2002; 12:640-7.

11. Fu J, Bian M, Jiang Q, Zhang C. Roles of Aurora kinases in mitosis and tumorigenesis. Mol Cancer Res. 2007; 5:1-10.

12. Lampson MA, Cheeseman IM. Sensing centromere tension: Aurora B and the regulation of kinetochore function. Trends Cell Biol. 2011; 21:133-40.

13. Cheeseman IM, Anderson S, Jwa M, Green EM, Kang JS, Yates Iii JR, Chan CSM, Drubin DG, Barnes G. Phosphoregulation of kinetochore-microtubule attachments by the Aurora kinase Ipl1p. Cell. 2002; 111:163-72.

14. Shao H, Huang Y, Zhang L, Yuan K, Chu Y, Dou Z, Jin C, Garcia-Barrio M, Liu X, Yao X. Spatiotemporal dynamics of Aurora B-PLK1-MCAK signaling axis orchestrates kinetochore bi-orientation and faithful chromosome segregation. Sci Rep. 2015; 5:12204.

15. Chu Y, Yao PY, Wang W, Wang D, Wang Z, Zhang L, Huang Y, Ke Y, Ding X, Yao X. Aurora B kinase activation requires survivin priming phosphorylation by PLK1. J Mol Cell Biol. 2011; 3:260-7.

16. Sessa F, Mapelli M, Ciferri C, Tarricone C, Areces LB, Schneider TR, Stukenberg PT, Musacchio A. Mechanism of Aurora B activation by INCENP and inhibition by Hesperadin. Mol Cell. 2005; 18:379-91.

17. Iimori M, Watanabe S, Kiyonari S, Matsuoka K, Sakasai R, Saeki H, Oki E, Kitao H, Maehara Y. Phosphorylation of EB2 by Aurora B and CDK1 ensures mitotic progression and genome stability. Nat Commun. 2016; 7:11117.

18. González-Loyola A, Fernández-Miranda G, Trakala M, Partida D, Samejima K, Ogawa H, Cañamero M, de Martino A, Martínez-Ramírez Á, de Cárcer G, Pérez de Castro I, 
Earnshaw WC, Malumbres M. Aurora B overexpression causes aneuploidy and p21Cip1 repression during tumor development. Mol Cell Biol. 2015; 35:3566-78.

19. Fernández-Miranda G, de Castro IP, Carmena M, AguirrePortolés C, Ruchaud S, Fant X, Montoya G, Earnshaw WC, Malumbres M. SUMOylation modulates the function of Aurora-B kinase. J Cell Sci. 2010; 123:2823-33.

20. Liu D, Davydenko O, Lampson MA. Polo-like kinase-1 regulates kinetochore-microtubule dynamics and spindle checkpoint silencing. J Cell Biol. 2012; 198:491-9.

21. Jang YJ, Lin CY, Ma S, Erikson RL. Functional studies on the role of the C-terminal domain of mammalian polo-like kinase. Proc Natl Acad Sci USA. 2002; 99:1984-9.

22. Lee KS, Grenfell TZ, Yarm FR, Erikson RL. Mutation of the polo-box disrupts localization and mitotic functions of the mammalian polo kinase Plk. Proc Natl Acad Sci U S A. 1998; 95:9301-6.

23. Hanisch A, Wehner A, Nigg EA, Silljé HHW. Different Plk1 functions show distinct dependencies on Polo-Box Domainmediated targeting. Mol Biol Cell. 2006; 17:448-59.

24. Kerppola TK. Design and implementation of bimolecular fluorescence complementation (BiFC) assays for the visualization of protein interactions in living cells. Nat Protoc. 2006; 1:1278-86.

25. Suijkerbuijk SJ, Vleugel M, Teixeira A, Kops GJ. Integration of kinase and phosphatase activities by BUBR1 ensures formation of stable kinetochore-microtubule attachments. Dev Cell. 2012; 23:745-55.

26. Maresca TJ, Salmon ED. Intrakinetochore stretch is associated with changes in kinetochore phosphorylation and spindle assembly checkpoint activity. J Cell Biol. 2009; 184:373-81.
27. Krenn V, Musacchio A. The Aurora B kinase in chromosome bi-orientation and spindle checkpoint signaling. Front Oncol. 2015; 5:225.

28. Herman JA, Toledo CM, Olson JM, DeLuca JG, Paddison PJ. Molecular pathways: regulation and targeting of kinetochore-microtubule attachment in cancer. Clin Cancer Res. 2015; 21:233-9.

29. Tauchman EC, Boehm FJ, DeLuca JG. Stable kinetochoremicrotubule attachment is sufficient to silence the spindle assembly checkpoint in human cells. Nat Commun. 2015; 6:10036.

30. Kang YH, Park JE, Yu LR, Soung NK, Yun SM, Bang JK, Seong YS, Yu H, Garfield S, Veenstra TD, Lee KS. Self-regulated Plk1 recruitment to kinetochores by the Plk1-PBIP1 interaction is critical for proper chromosome segregation. Molecular Cell. 2006; 24:409-22.

31. Petronczki M, Lenart P, Peters JM. Polo on the rise-from mitotic entry to cytokinesis with Plk1. Dev Cell. 2008; 14:646-59.

32. Goto H, Kiyono T, Tomono Y, Kawajiri A, Urano T, Furukawa K, Nigg EA, Inagaki M. Complex formation of Plk1 and INCENP required for metaphase-anaphase transition. Nat Cell Biol. 2006; 8:180-7.

33. Lee HS, Park YY, Cho MY, Chae S, Yoo YS, Kwon $\mathrm{MH}$, Lee CW, Cho H. The chromatin remodeller RSF1 is essential for PLK1 deposition and function at mitotic kinetochores. Nat Commun. 2015; 6:7904. 